\title{
Data-Dependent Jitter in Serial Communications
}

\author{
Behnam Analui, Student Member, IEEE, James F. Buckwalter, Student Member, IEEE, and Ali Hajimiri, Member, IEEE
}

\begin{abstract}
We present a method for predicting data-dependent jitter (DDJ) introduced by a general linear time-invariant LTI system based on the system's unit step response. We express the exact DDJ of a first-order system and verify the validity of the solution experimentally. We then propose a perturbation technique to generalize the analytical expression for DDJ. We highlight the significance of the unit step response in characterizing DDJ and emphasize that bandwidth is not a complete measure for predicting DDJ. We separate the individual jitter contributions of prior bits and use the result to predict the DDJ of a general LTI system. In particular, we identify a dominant prior bit that signifies the well-known distribution of deterministic jitter, the two impulse functions. We also show a jitter minimization property of high-order LTI systems. We verify our generalized analytical expression of DDJ for several real systems including an integrated CMOS 10-Gb/s trans-impedance amplifier by comparing the theory and measurement results. The theory predicts the jitter with as low as only $7.5 \%$ error.
\end{abstract}

Index Terms-Data-dependent jitter (DDJ), deterministic jitter (DJ), jitter, jitter distribution, linear time-invariant (LTI) system, step response.

\section{INTRODUCTION}

H IGH-SPEED serial communications depends upon phenomenological understanding of timing jitter. Timing jitter of data transition is deviations of the threshold-crossing time, i.e., time at which data crosses a decision threshold, compared to a reference clock. The transmitter, channel, and receiver contribute to timing jitter of the data sequence. In addition, the timing jitter of the data is inherited as phase uncertainty of the recovered sampling clock. The bit error rate (BER) of the regenerated data sequence in the receiver is degraded by the timing jitter of the data and sampling clock. Nonidealities such as bandwidth limitation and medium dispersion exacerbate jitter effects.

Data timing jitter is separated into random jitter (RJ) and deterministic jitter (DJ) [1]. RJ is random variations of thresholdcrossing time often due to a noise source, e.g., in the synchronizing clock [2]. DJ is further categorized into data-dependent jitter (DDJ), duty cycle distortion jitter, and bounded uncorrelated to data jitter (e.g., crosstalk jitter or sinusoidal jitter) [1]. DDJ is threshold-crossing time deviations correlated to the previous bits on the current data bit. It is also known as pattern jitter.

Manuscript received April 1, 2005; revised July 7, 2005. This work was supported in part by the National Science Foundation and by the Lee Center for Advanced Networking, California Institute of Technology.

B. Analui was with the High-Speed Integrated Circuits Group, California Institute of Technology, Pasadena, CA 91125 USA. He is now with Luxtera Inc., Carlsbad, CA 92008 USA (e-mail: behnam@luxtera.com).

J. F. Buckwalter and A. Hajimiri are with the High-Speed Integrated Circuits Group, California Institute of Technology, Pasadena, CA 91125 USA (e-mail: jamesb@caltech.edu; hajimiri@caltech.edu).

Digital Object Identifier 10.1109/TMTT.2005.857118
DDJ is often caused by bandwidth limitations of the system or electromagnetic reflections of the signal. Therefore, DDJ has a larger impact on high-speed transmission systems with restricted bandwidth. In this study, we propose methods for characterizing DDJ theoretically based on system parameters.

The impact of timing jitter on the performance of different communication links has been studied extensively [3]-[10]. However, these studies have focused on the effect of digital pattern on the output jitter of the extracted clock. They have neglected the limitations of all other blocks in the communication link. For instance, Byrne et al. have investigated the accumulation effect of timing jitter in a series of regenerators with special attention to the effect of pattern jitter [4]. However, the analysis is limited to a simple second-order tank as the timing extraction block. Saltzberg has estimated the aggregate effect of RJ and DDJ using Taylor series expansion and has calculated the jitter of the extracted sampling clock [5]. Similarly, Gardner has compared the effect of pattern jitter on different clock recovery schemes [7]. He has presented a relation between DDJ and the sampling clock phase variation with qualitative explanations. Huang et al. have proposed pulse shapes that result in DDJ-free data streams [8]. However, they have emphasized the peak-to-peak DDJ and have calculated it from the two data sequences that result in the maximum shift of the threshold-crossing time. They have assumed a given form for the received data stream, namely, an ideal noncausal Nyquist pulse. All these studies condition the system that generates DDJ to several assumptions. A model for the DDJ generated from a general linear time-invariant (LTI) system is still lacking.

In a different context, jitter modeling techniques are developed for separating and measuring jitter performance of devices in communication links [1], [10]-[12]. Reliable jitter measurement methods are more important in high-speed devices, where bandwidth limitations aggravate DDJ. Therefore, predicting DDJ contribution is essential to accurate measurement systems. For instance, Shimanouchi has related the bandwidth of an automatic test equipment (ATE) system and the DDJ [10]. However, his analysis was based on the previous data transition only. In addition, he limits the model to first-order system response.

Although the significance of DDJ has been realized in the aforementioned literature, theoretical analysis of DDJ and study of its relation to system parameters such as bandwidth has been neglected. The main contribution of this paper is to expand the study of [13] and propose a method for predicting DDJ for a general LTI system in a context suitable for circuits and system designers. The dependence of DDJ on system parameters provides additional insights for minimizing jitter and highlights that increasing the bandwidth does not necessarily minimize DDJ. In 
addition, the technique reduces the simulation or measurement time remarkably by relating DDJ characterization linearly to the number of prior bits. The conventional computation grows exponentially with the number of bits because it requires passing all possible sequences through the system. The theoretical results are matched with jitter histogram measurements.

In this paper, we first define DDJ formally in Section II. In Section III, an analytical expression for DDJ is derived for firstorder LTI systems. The expressions are associated to commonly used distribution of DDJ and the results are experimentally verified. Next, we generalize the analysis for any LTI system with a known step response in Section IV. A perturbation method is introduced that approximates DDJ by separating the jitter contributions of previous bits. In Section V, we compare the measured DJ of real communication media with analytical expressions of Section IV and demonstrate that the presented analytical results estimate DDJ accurately and are reliable for predicting jitter.

\section{FRAMEWORK}

\section{A. Data Jitter}

A typical serial communication receiver regenerates data by sampling the received signal. Sampling occurs synchronous to a clock extracted from the corrupted data. Ideally, the sampling clock should occur between adjacent data transitions to optimize the BER. For a given symbol rate, each threshold crossing time ideally occurs at integer multiples of the symbol period. However, it deviates from the ideal value due to several factors in the link (e.g., noise, limited channel bandwidth, limited receiver front-end bandwidth). Consequently, the knowledge of the effect of the system on data threshold crossing times and the sampling clock timing is essential for optimizing BER.

Data jitter is the deviation of the data threshold crossing times from a reference time. The total jitter is modeled as the sum of two independent random variables, RJ, $\Delta t_{\mathrm{rj}}$, and DJ, $\Delta t_{\mathrm{dj}}[1]$ as follows:

$$
\Delta t_{\mathrm{tj}}=\Delta t_{\mathrm{rj}}+\Delta t_{\mathrm{dj}}
$$

Hence, the total jitter probability distribution function (PDF) is the convolution of the PDF of RJ and DJ [14]

$$
f_{\mathrm{tj}}(\Delta t)=f_{\mathrm{rj}}(\Delta t) \otimes f_{\mathrm{dj}}(\Delta t)
$$

where $f(\Delta t)$ is the PDF of each jitter term.

$\mathrm{RJ}$ is modeled by a Gaussian random variable [1]. DJ has systematic origins such as bandwidth limitation or crosstalk. In general, it has stochastic characteristics because transmitted data or data in neighboring channels is random. Efforts for modeling the PDF of DJ are typically based on results from measurement techniques and numerical computation algorithms [12]-[16]. The distribution function of DJ has been previously modeled as two impulse functions [1], [15]. DJ is characterized by the distance between the two impulses. ${ }^{1}$ Fig. 1(a) illustrates how total jitter distribution results from the combination of RJ and DJ. Fig. 1(b) shows a typical measurement result for

\footnotetext{
${ }^{1}$ Wavecrest Technol., Eden Prairie, MN. Jitter Fundamentals. [Online]. Available: http://www.wavecrestcorp.com/technical/pdf/jittfun_hires_sngls.pdf
}

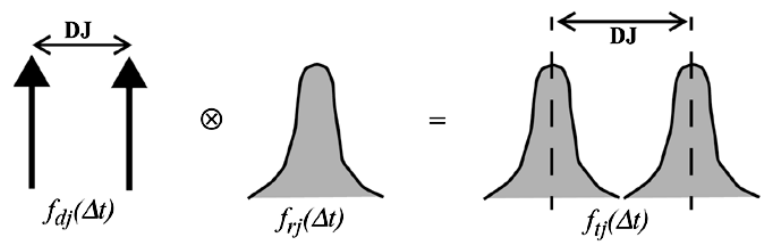

(a)

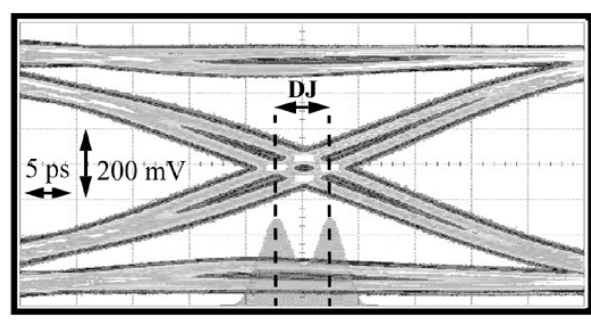

(b)

Fig. 1. (a) Distribution of total jitter from the convolution of RJ and DJ PDFs. (b) Eye diagram and jitter histogram measurement for a data sequence passed through a microstrip transmission line on FR4 PCB.

the eye diagram of a received data sequence around threshold crossing time. The measured jitter histogram approximates data jitter distribution in Fig. 1(a). In this study, we analytically explore DDJ, one of the major components of DJ. We propose methods for characterizing DDJ theoretically based on system parameters. Analytical studies on other sources of DJ can be found in [17].

\section{B. $D D J$}

DDJ is the deviation of each data threshold-crossing time from a reference time due to the residual memory of data bits. Limited bandwidth of the transmission medium (e.g., printed circuit board (PCB) traces), receiver front-end [e.g., trans-impedance amplifier (TIA)], or electromagnetic reflections cause prior symbols to interfere with the current transition. While the effect of inter-symbol interference (ISI) on the amplitude of the received symbols has been studied (e.g., [18] and [19]), its effect on the timing needs further analysis. The effect of ISI on timing is to change the threshold-crossing time of a data transition and cause DDJ.

To find DDJ, a sequence of random binary data is passed through an LTI system that models the overall response of the band-limited link and causes DDJ. The last two bits of the sequence are either " 01 " or " 10 " to model a rising edge transition or falling edge transition, respectively. The variation of the crossing time of the transition can be related to the data statistics to calculate DDJ. The process is illustrated in Fig. 2. For symmetric input rising and falling transitions and a threshold of half signal swing, the jitter distributions for rising and falling transitions are identical and the calculation of one is sufficient.

A random data sequence arriving at the input can be represented by

$$
\begin{aligned}
& x(t)=u(t)+\sum_{k=-\infty}^{-2} a_{k} \cdot p_{i}\left(t-k T_{b}\right), \quad a_{k} \in\{0,1\} \\
& p_{i}(t)= \begin{cases}1, & 0 \leq t \leq T_{n} \\
0, & \text { otherwise }\end{cases}
\end{aligned}
$$




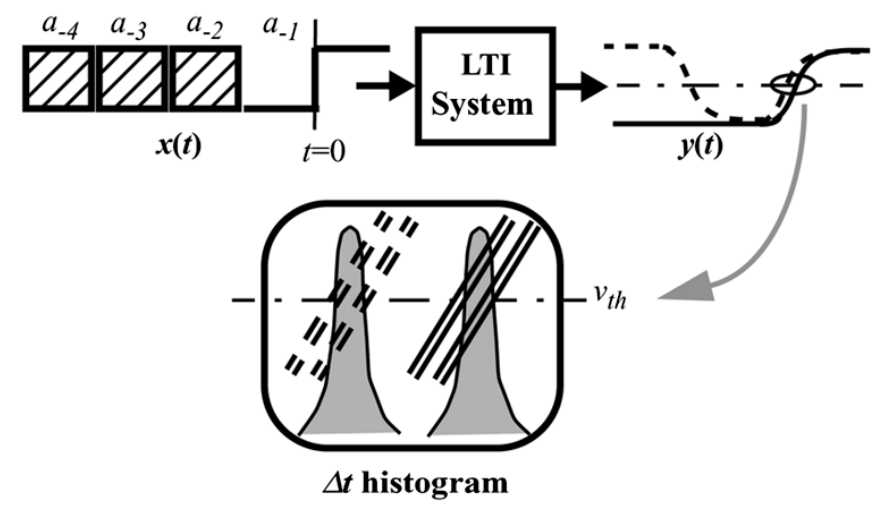

Fig. 2. Response of a general LTI system to a random bit sequence and generation of DDJ.

where $u(t)$ is the unit step function and models the rising edge. $p_{i}(t)$ is the unit pulse signal, as described in (4), with duration of bit period $T_{b} . a_{k} s$ are the random bits that are either " 1 " or "0" with a given probability. The sum in (3) starts from $k=-2$ to guarantee a rising edge at $t=0$. The output of the system can be written as

$$
y(t)=s(t)+\sum_{k-\infty}^{-2} a_{k} \cdot p_{o}\left(t-k T_{b}\right)
$$

where $s(t)$ and $p_{o}(t)$ are, respectively, the system step response and unit pulse response.

The solution to

$$
y\left(t_{c}\right)=v_{\mathrm{th}}=0.5
$$

for $t_{c}$ is the time of the threshold-crossing event as a function of data statistics and system parameters. We compare $t_{c}$ to the time of threshold-crossing event when all the $a_{k} s$ are zero and we denote it by $t_{0}$. We can calculate $t_{0}$ by solving $s\left(t_{0}\right)=v_{\text {th }}=$ 0.5 .

DDJ is then defined as

$$
\Delta t \equiv t_{0}-t_{c}
$$

We will solve (6) for the first-order system as an example in Section III and analyze the general LTI system in Section IV.

\section{ANALYTICAL EXPRESSION FOR DDJ: FIRST-ORDER SYSTEM}

\section{A. Analytical Expression for Threshold Crossing Time}

Here, we analyze the DDJ of a first-order system as described by the transfer function

$$
H(s)=\frac{1}{1+\tau s} .
$$

Here, $\tau$ is the system time constant, and the associated $3-\mathrm{dB}$ bandwidth is $1 /(2 \pi \tau)$. From (6) and (7), we can derive the DDJ random variable for a first-order system as

$$
\Delta t=-\tau \cdot \ln \left(1-\left(\frac{1-\alpha}{\alpha}\right) \sum_{k=-\infty}^{-2} a_{k} \cdot \alpha^{-k}\right)
$$

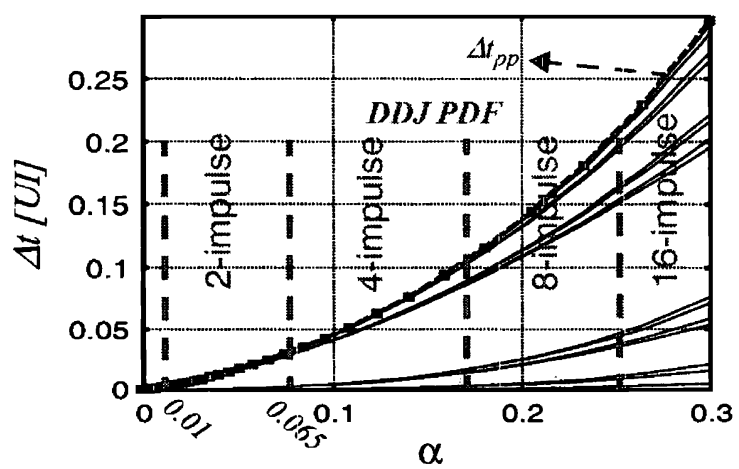

Fig. 3. Ensemble of normalized DDJ values for different ratios of bandwidth to bit rate along with the appropriate model to use for DDJ PDF.

where we define $\alpha \equiv e^{-T_{b} / \tau}$ that relates the system bandwidth and bit rate. In a system with a large bandwidth compared to the input data rate, $\alpha$ approaches zero. On the other hand, if the bandwidth is small, the data transitions take longer. The upper limit on $\alpha$ for this calculations is set if we assume the rising transition crosses the threshold within a bit period. This forces $\alpha$ to values smaller than 0.5 . At $\alpha=0.5$, the bandwidth is only $11 \%$ of the bit rate. ${ }^{2}$

Equation (9) relates the impact of each prior bit and the threshold-crossing time deviation. For any data transition, the prior bits are random sequences that overall result in an ensemble of $\Delta t$ values. As $\alpha \leq 0.5$, the more recent bits have a dominant effect on jitter and $a_{-2}$ has the largest impact. The residual effect of the bits also vanishes exponentially for larger system bandwidth to bit rate ratio, i.e., when $\alpha$ approaches zero. Fig. 3 captures these effects by plotting $\Delta t$ in unit intervals (UIs) for different values of $\alpha$. For each $\alpha$, all the possible values of $\Delta t$ are plotted. We include the impact of four prior bits and neglect the effect of more distant bits. A larger $\alpha$ corresponds to smaller bandwidth to bit rate ratio causing divergence in $\Delta t$ values and larger DDJ. If we change the scale on the $x$-axis and plot DDJ for small values of $\alpha$, we will observe similar DDJ characteristics on a different scale of $\Delta t$. In fact, it can be seen from (9) that, for each data sequence, $\Delta t$ takes a unique value. Therefore, on a smaller scale, for $\alpha$, the same divergence characteristics would be observed for $\Delta t$ values. In fact, $\Delta t$ has a self-similar behavior for different scales of $\alpha$.

For $001 \leq \alpha \leq 0.065, \Delta t$ is concentrated around two values. In this range of system bandwidth, the DDJ distribution can be modeled with two impulses that carry the probability weight expressed in Section II. However, for larger $\alpha$, the distribution should be extended to four or more impulses, as can be seen from Fig. 3. In a first-order system, the concentration of data jitter around two values corresponds to bandwidth range, where only the penultimate bit $a_{-2}$ has a remarkable effect on jitter. Since $a_{-2}$ is " 1 " or " 0 ," the data jitter is divided into two mean probability masses, modeled by the two impulse functions. Similar behavior for DDJ distribution is generalized to higher order systems, as will be seen in Section IV. A dominant prior bit (not necessarily $a_{-2}$ ) will be identified that shapes DDJ distribution as two impulse functions.

${ }^{2}$ In a practical communication link, the receiver bandwidth is typically $70 \%-80 \%$ of the bit rate. 


\section{B. Peak-to-Peak Jitter}

DDJ is bounded. It can be characterized by its peak-to-peak jitter value $\Delta t_{\mathrm{pp}}$. From (9), extremes of $\Delta t$ are obtained for all one and all zero prior bits. The all zero prior bits sequence corresponds to the latest threshold crossing time, which is also selected as the reference time $t_{0}$. Therefore, we can calculate peak-to-peak DDJ as

$$
\Delta t_{\mathrm{pp}}=-\tau \cdot \ln (1-\alpha)
$$

which is overlaid with a dashed line in the plot of Fig. 3. Since the latest crossing time is referenced, the plot shows that $\Delta t_{\mathrm{pp}}$ sets an upper bound on $\Delta t$.

\section{Scale-One DDJ}

In modern serial communication links, measured total jitter distributions resemble the jitter histogram in Fig. 1(b). In such systems, a useful measure of DDJ is the distance between the two impulse functions in Fig. 1(a) or the separation between the means of the two Gaussian distributions. According to discussions in Section III-A, the two impulse distribution results when the impact of only one prior bit $\left(a_{-2}\right)$ on jitter is included. Therefore, we define the separation of the impulses as follows and call it the scale-one DDJ DDJ : $_{1}$

$$
\mathrm{DDJ}_{1}=E\left\{\Delta t \mid a_{-2}=0\right\}-E\left\{\Delta t \mid a_{-2}=1\right\}
$$

where $E\{\cdot\}$ is the conditional expected value of $\Delta t$ given $a_{-2}$. For equal probabilities of " 1 " and " 0 ," we can show

$$
\mathrm{DDJ}_{1}=\frac{\tau}{2} \ln \frac{1+\alpha}{1-\alpha+\alpha^{2}} .
$$

We verified the expression in (12) experimentally by testing a first-order $R C$ filter that serves as the first-order system [20]. A $2^{7}-1$ pseudorandom bit sequence (PRBS) was applied to the filter and the jitter histogram was measured using Agilent's 86100 communication analyzer. The input bit rate was scanned over a wide range of observable $\mathrm{DDJ}_{1}$ values. An example histogram is shown in Fig. 4(a) for $\alpha=0.1$ and shows significant amount of $\mathrm{DDJ}_{1}$. The separation of the jitter mean of the two Gaussians in the histogram was measured. Fig. 4(b) demonstrates excellent agreement between (12) and the measurement results. For $\alpha<0.02$, RJ dominated $\mathrm{DDJ}_{1}$.

\section{DDJ ANALYTICAL EXPRESSION: GENERAL LTI SYSTEM}

\section{A. Perturbation Method}

For a general LTI system, (6) may not be solvable analytically. We propose a technique that approximates DDJ for a general LTI system based only on its step response. The method can be exploited easily in simulation or measurement to characterize DDJ and optimize jitter performance.

DDJ occurs because the tails of prior bits perturb the time that the data transition crosses the threshold level. In the absence of any prior bit, threshold crossing time is $t_{0}$, as discussed in Section II. However, if $a_{k}$ is 1 , the $k$ th prior bit changes $s(t)$ by $p_{o}\left(t_{0}-k T_{b}\right)$ in (5). The perturbation shifts the threshold crossing time from $t_{0}$ and causes jitter. Assuming

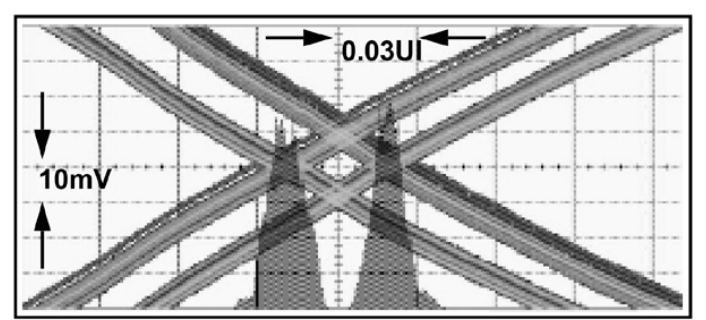

(a)

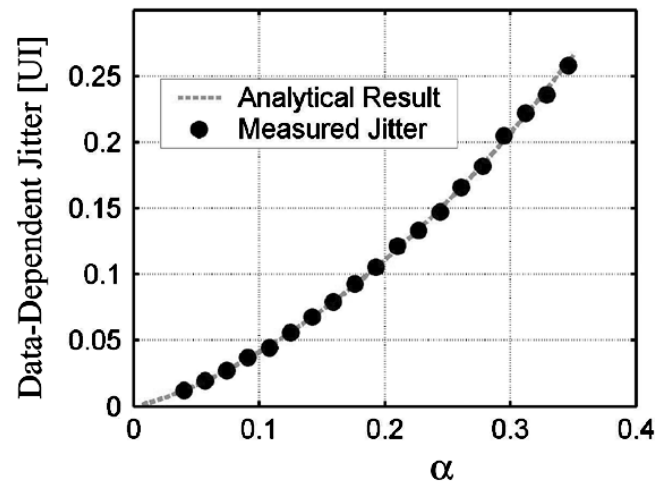

(b)

Fig. 4. (a) Eye diagram magnified around threshold crossing time for a first-order system at $\alpha=0.1$. (b) Comparison of the measurement results for $\mathrm{DDJ}_{1}$ and the analytical expression in (12) for a first-order system.

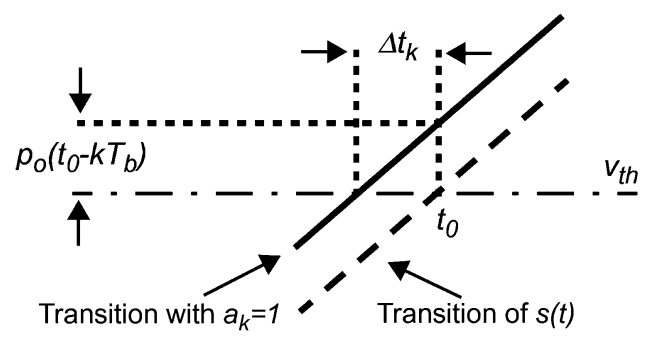

Fig. 5. Deviation of the threshold-crossing time due to the effect of the $k$ th bit.

$p_{o}\left(t_{0}-k T_{b}\right) \ll s\left(t_{0}\right)$, the shift in threshold crossing time from the contribution of the $k$ th bit can be calculated from the slope of $s(t)$ at $t_{0}$ and the shift in the amplitude of $s(t)$. This process is shown graphically in Fig. 5. The threshold crossing time shift due to the $k$ th bit is denoted by $\Delta t_{k}$. We have

$$
\Delta t_{k} \cong \frac{p_{o}\left(t_{0}-k T_{b}\right)}{\left.\frac{d s(t)}{d t}\right|_{t=t_{0}}}
$$

and the overall perturbation effect (DDJ) is defined as

$$
\Delta t \equiv \sum_{k=-\infty}^{-2} a_{k} \Delta t_{k}=\frac{-1}{\left.\frac{d s(t)}{d t}\right|_{t=t_{0}}} \cdot \sum_{k=-\infty}^{-2} a_{k} p_{o}\left(t_{0}-k T_{b}\right)
$$

This technique is based on classical perturbation theory (e.g., [21]). The assumption made above on the amount of perturbation bounds the accuracy of the method. In a practical system, the bandwidth is chosen such that unit pulse response fall time is within $T_{b}$. Therefore, $p_{o}\left(t_{0}-k T_{b}\right)$ is much smaller than $v_{\text {th }}$ and 
(a)

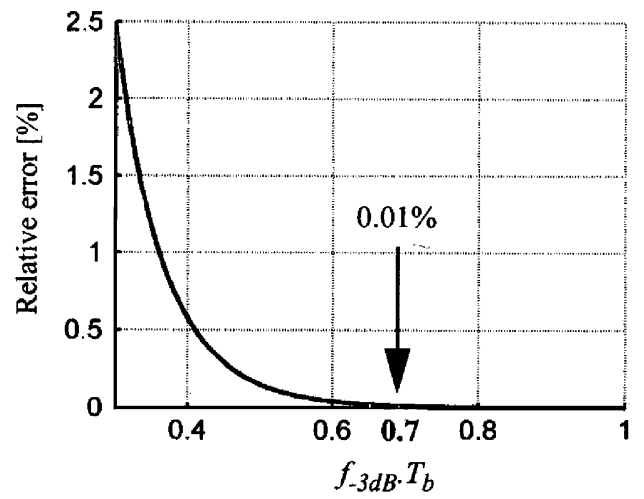

(b)

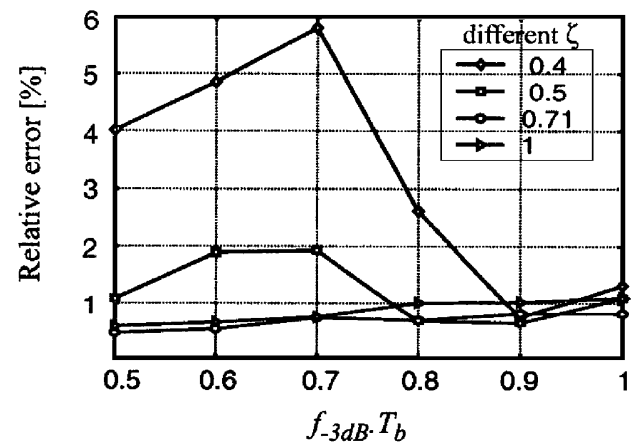

Fig. 6. Worst case accuracy of the perturbation method in predicting DDJ (a) For a first-order system. (b) For a second-order system.

(14) is a good approximation. If the link is designed such that the received pulse has the shape of a raised cosine signal, the approximation still holds. For such pulses, the residual memory of prior bits changes slowly around the threshold crossing [22]. Therefore, the perturbation of the step response is $p_{o}\left(t_{0}-k T_{b}\right)$. A similar methodology was used to calculate the reference jitter in a clock recovery system [5], [23], [24].

We evaluated the results in (14) for all possible bit sequences and compared them against the accurate DDJ in (9) for a firstorder system. We limit $k$ to $-10 \leq k \leq-2$ to account for the 11 most recent bits only because the effect of the bits exponentially decreases. Error in DDJ prediction is calculated for each bit sequence at different ratios of bandwidth $(1 / 2 \pi \tau)$ to bit rate $\left(1 / T_{b}\right)$ and, for each ratio, the worst case relative error is plotted in Fig. 6(a). The perturbation method approximation has worst case accuracy of better than $2.5 \%$ in a practical range of bandwidth. Moreover, at the nominal bandwidth to bit rate ratio of 0.7 , the error is only $0.01 \%$. For a first-order system, the error in approximation is identical even if $-3 \leq k \leq-2$. Therefore, (14) introduces a basis for a very efficient technique of calculating DDJ.

A further verification of the perturbation technique is done for an all-pole second-order system with transfer function

$$
H(s)=\frac{\omega_{n}^{2}}{s^{2}+2 \zeta \omega_{n} s+\omega_{n}^{2}}
$$

where $\omega_{n}$ is the natural frequency and $\zeta$ is the damping factor. The exact DDJ value for this system is computed from MATLAB simulations of system output for all possible bit sequences. The approximated DDJ is then calculated using (14). The results are compared and the worst case relative error is plotted in Fig. 6(b) for different damping factors over a practical range of bandwidth normalized to bit rate. Again, small relative errors verify that (14) is an accurate expression for predicting the DDJ of a general LTI system based on its step response.

\section{B. Peak-to-Peak Jitter and Scale-One DDJ}

We can use (14) to estimate the peak-to-peak DDJ for a general LTI system. We have

$$
\Delta t_{\mathrm{pp}}=\max \{\Delta t\}-\min \{\Delta t\} .
$$

The maximum of $\Delta t$ is achieved for the data sequence in which $a_{k}=1$ if $p_{0}\left(t_{0}-k T_{b}\right) \leq 0$ and $a_{k}=0$ otherwise. Similarly, the minimum of $\Delta t$ is achieved for the data sequence, where $a_{k}=1$ if $p_{o}\left(t_{0}-k T_{b}\right) \leq 0$ and $A_{K}=0$ otherwise. Therefore, (16) is simplified to

$$
\Delta t_{\mathrm{pp}}=\frac{1}{\left.\frac{d s(t)}{d t}\right|_{t=t_{0}}} \cdot \sum_{k=-\infty}^{-2}\left|p_{o}\left(t_{0}-k T_{b}\right)\right| .
$$

Scale-one DDJ can also be defined for a general LTI system similar to (11). However, the predominant impact on jitter is not necessarily related to $a_{-2}$, as discussed in Section III. The pulse response of the system and the bit rate determine the effect of prior bits. The effect of each prior bit can be estimated separately from (13) and the bit with the most prominent impact can be distinguished. Using the same definition as in (11) and assuming that $a_{m}$ has the largest impact on DDJ, then

$$
\begin{aligned}
\mathrm{DDJ}_{1} & =\left|E\left\{\Delta t \mid a_{m}=0\right\}-E\left\{\Delta t \mid a_{m}=1\right\}\right| \\
& =\left|E\left\{\sum_{\substack{k=-\infty \\
k \neq m}}^{-2} a_{k} \Delta t_{k}\right\}-E\left\{\Delta t_{m}+\sum_{\substack{k=-\infty \\
k \neq m}}^{-2} a_{k} \Delta t_{k}\right\}\right| \\
& =\left|\Delta t_{m}\right| .
\end{aligned}
$$

Therefore, we conclude

$$
\mathrm{DD} J_{1}=\left|\frac{p_{o}\left(t_{0}-m T_{b}\right)}{\left.\frac{d s(t)}{d t}\right|_{t=t_{0}}}\right|
$$

which is an important yet simple expression that determines the separation of the two impulses in the PDF of DDJ, as in Fig. 1(a), for a general LTI system. It can be integrated into any communication link design or circuit design simulation software to predict the DDJ contribution of the corresponding component in the system. In addition, $\mathrm{DDJ}_{1}$ can be easily measured using a general-purpose high-speed oscilloscope. We will verify (19) experimentally in Section V.

A significant advantage of the perturbation method is the remarkable reduction of the simulation or measurement time of DDJ. In fact, simulation time for peak-to-peak DDJ is now linearly related to $k$, while direct calculation from (6) requires passing all the $2^{k}$ possible sequences through the system, which increases exponentially with $k$. 
(a)

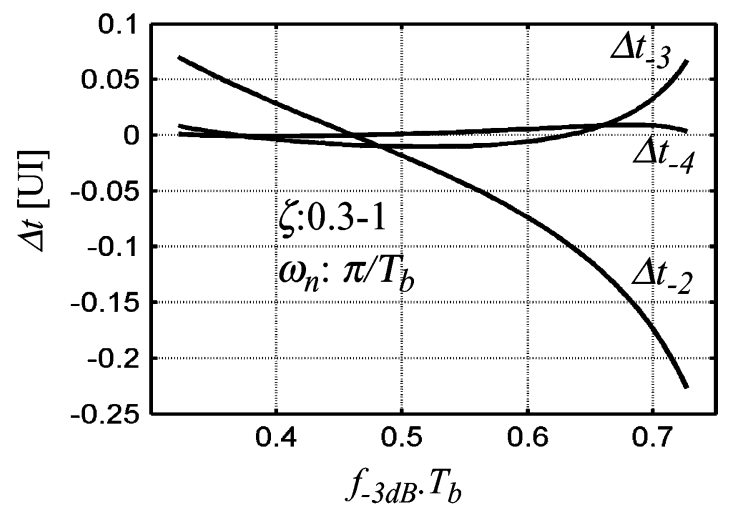

(b)

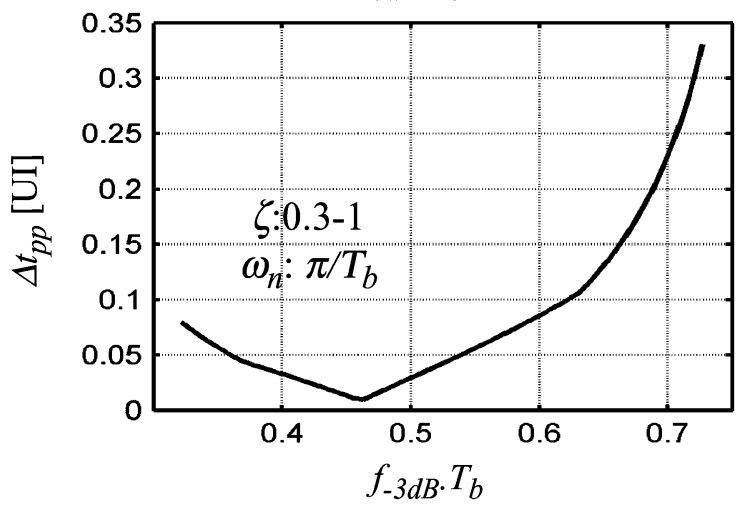

Fig. 7. (a) Variation of the impacts of the last three prior bits on DDJ in a second-order system. (b) Existence of a minimum in the peak-to-peak DDJ.

\section{DDJ Minimization}

In a first-order system, any $a_{k}=1$ will increase the absolute value of DDJ. Furthermore, the closer the bit to the data transition, the stronger its impact on data jitter. However, this is not generally true for all LTI systems. It can be seen from (13) that the sign and value of $\Delta t_{k}$ depends on $p_{o}\left(t_{0}-k T_{b}\right)$ and, based on the response of the system, the effect of each prior bit can dramatically vary independent of the other bits. The pulse response in (13) is particularly sampled at integer multiples of bit period. Therefore, for a given bit rate, the system can be designed such that its pulse response reduces dominant DDJ terms and minimizes overall jitter. Pulse shapes that result in minimum jitter in addition to minimum ISI in the receiver have been studied [8], [25]. As an example, the variations of the first three DDJ terms from (13) is plotted in Fig. 7(a) for a second-order system with different bandwidth to bit-rate ratios. The selected range covers under-damped, over-damped, and critically damped systems. In the range of $0.46-0.48$ for the normalized bandwidth, $\Delta t_{-3}$ has a larger impact than $\Delta t_{-2}$. In addition, there exist a minimum in the peak-to-peak DDJ, as illustrated in Fig. 7(b). This jitter minimization behavior can be observed in higher order systems as well. An experimental example is shown in Fig. 8, where the output eye diagram of a 4-in copper microstrip transmission line on a conventional FR4 board is plotted at two different bit rates. The peak-to-peak jitter is clearly larger at the lower bit rate. As will be illustrated in Section V, increasing the bandwidth blindly does not necessarily reduce the DDJ.

\section{EXPERIMENTAL VERIFICATION}

Equation (19) provides a simple means for finding the DDJ contributions of any LTI system for any bit rate based only on the

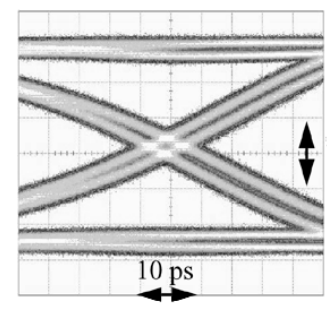

(a)

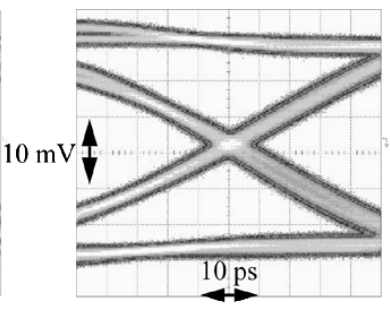

(b)
Fig. 8. Output eye diagram of a 4-in microstrip line on FR4 PCB at: (a) 5 and (b) $6.5 \mathrm{~Gb} / \mathrm{s}$ demonstrates larger peak-to-peak DJ at lower bit rate.

TABLE I

MEASURED AND ANALYTICAL DDJ $\mathrm{J}_{1}$

\begin{tabular}{|c|c|c|c|c|c|}
\hline DUT & Bit Rate & $\begin{array}{c}\text { Measured } \\
D D J_{1}[\text { psec] }\end{array}$ & Bit & $\begin{array}{l}\text { Predicted } \\
\Delta t_{k}[\text { psec] }\end{array}$ & Error \\
\hline ZFL-1000 & $1.3 \mathrm{~Gb} / \mathrm{s}$ & 7.665 & $a_{-2}$ & 7.15 & $6.7 \%$ \\
\hline Microstrip & $10 \mathrm{~Gb} / \mathrm{s}$ & 5.35 & $a_{-3}$ & 5.23 & $2.3 \%$ \\
\hline HP Filter & $1.2 \mathrm{~Gb} / \mathrm{s}$ & 20.5 & $a_{-2}$ & 18.96 & $7.5 \%$ \\
\hline BNC cable & $3 \mathrm{~Gb} / \mathrm{s}$ & 4.6 & $a_{-2}$ & 4.72 & $2.5 \%$ \\
\hline
\end{tabular}

step response. The pulse response can be stated in terms of step response as $p(t)=s(t)-s\left(t-T_{b}\right)$. We will verify the validity of the results experimentally by comparing the predictions of (19) with measured $\mathrm{DDJ}_{1}$ of several high-frequency systems including an integrated CMOS TIA. We associate $\mathrm{DDJ}_{1}$ to the separation of the means of two Gaussian distributions, as in Fig. 1(b), when the jitter histogram at the output of the device-under-test (DUT) is measured. We use Anritsu's MP1763C pulse pattern generator to provide the step input and PRBS input of the length $2^{7}-1$. We also use Agilent's 86100 communication analyzer to measure the step response and jitter histogram at the output. For each system, we first measure and record the step response. We then apply a PRBS at the input with varying bit rate. We measure $\mathrm{DDJ}_{1}$ at a bit rate that the system shows having a significant amount of DDJ. The bit rate is always such that data spectrum does not exceed the system bandwidth. This fact demonstrates that, while the system bandwidth is large enough to minimize amplitude distortion, DDJ still persists. The jitter histogram is measured after at least 500000 crossing events are captured by the oscilloscope. At the same time, we compute the pulse response from the measured step response and the current bit rate and calculate $\mathrm{DDJ}_{1}$ from (19). Finally, we compare the measured and analytically calculated $\mathrm{DDJ}_{1}$.

\section{A. Discrete Systems}

In one set of experiments, we carry out the procedure for various off-the-bench systems available in the laboratory. They include a Mini Circuit ZFL 1000-LN driver amplifier with 1-GHz bandwidth, a 9-in-long 50- $\Omega$ copper microstrip on standard FR4 PCB, a 10.5-in-long standard BNC coaxial cable, and an HP 11688A microwave high-order low-pass filter with cutoff frequency of $f_{c}=2.8 \mathrm{GHz}$. None of these systems has a simple first-order response. Therefore, the $\mathrm{DDJ}_{1}$ should be estimated from (19). The measurement results are summarized in Table I. 
Mini Circuit ZFL-1000
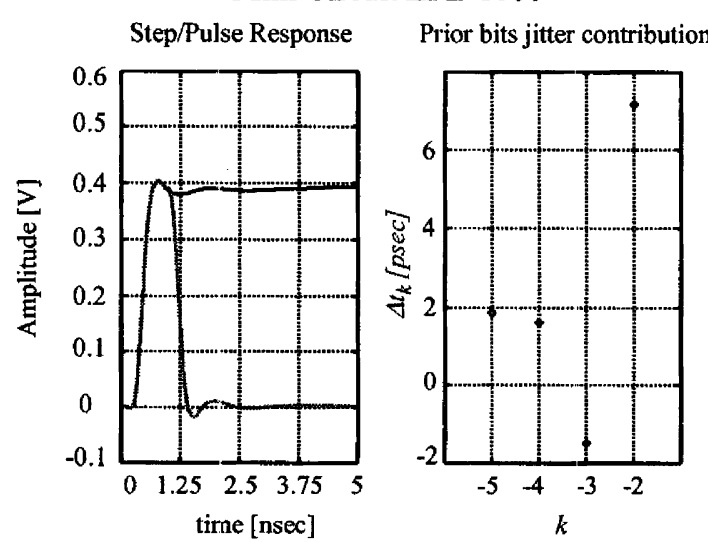

(a)

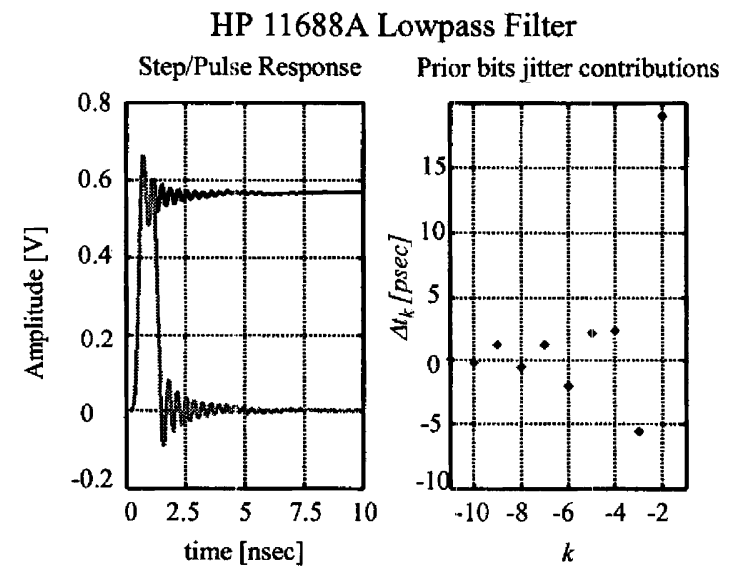

(c)
FR4 Copper Microstrip

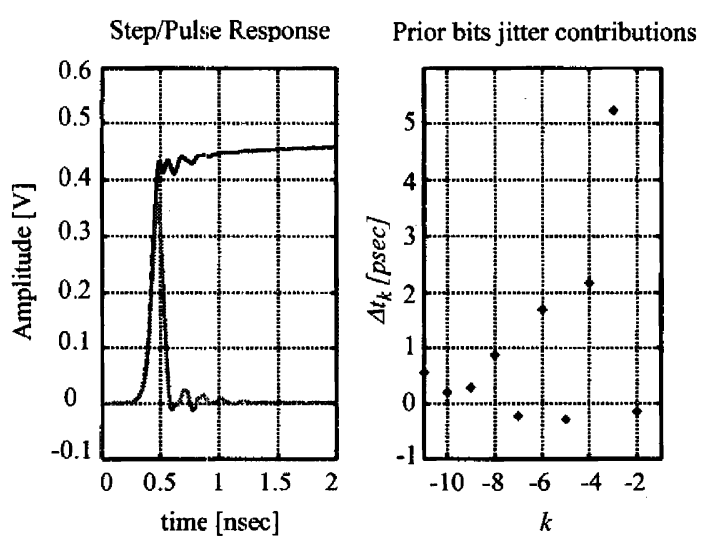

(b)

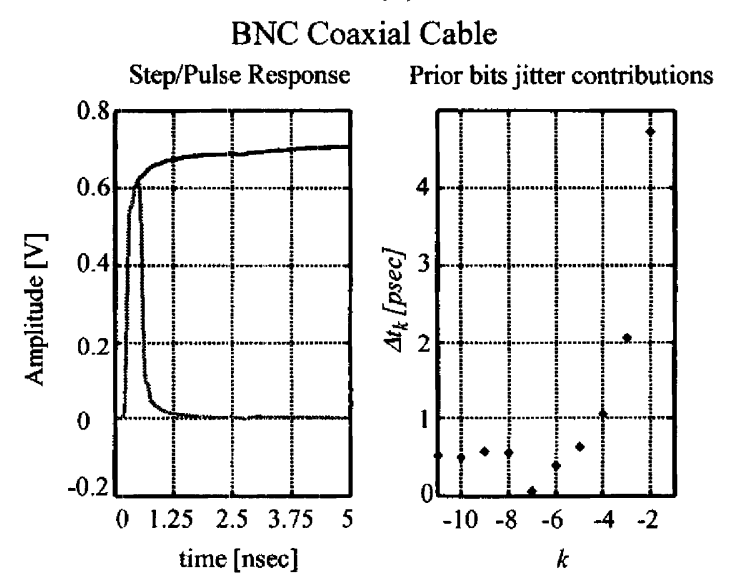

(d)

Fig. 9. Step response, pulse response, and individual jitter contributions of prior bits as calculated from (13) for the systems under test. (a) Mini Circuit ZFL-1000 amplifier. (b) Copper microstrip line on FR4 PCB. (c) HP 11688A low-pass filter. (d) BNC coaxial cable.

Small relative errors in the last column verify the validity of the analytical results for predicting DDJ. For the microstrip line, $a_{-3}$ rather than $a_{-2}$ has the most dominant effect on DDJ and causes the scale-one separation of the threshold crossing times.

Step response, pulse response, and the jitter contributions of some prior bits are plotted in Fig. 9 for the systems we tested. $\Delta t_{k}$ is calculated from a pulse response using (13). An important observation is the significance of the pulse-response shape of the system and its impact on DDJ at the output. HP 11688A is a low-pass filter with the $3-\mathrm{dB}$ cutoff frequency at $2.8 \mathrm{GHz}$. Comparing to ZFL-1000, an amplifier with 3-dB bandwidth of $1 \mathrm{GHz}$, one may suspect the DDJ contribution to overall jitter is larger for the amplifier due to smaller bandwidth. However, around the same bit rate $(1.2-1.3 \mathrm{~Gb} / \mathrm{s})$, the filter has significantly larger DDJ. This can be associated to the pulse response characteristics of the two systems, as illustrated in Fig. 9(a) and (c). Pulse response of the filter has larger ringing in its damping tail that dramatically increases the jitter from (13) because the samples of the pulse response at the measurement bit rate $(1.2 \mathrm{~Gb} / \mathrm{s})$ collide with the maxima and minima of the oscillating tail. Consequently, the contributions of prior bits are all significant and oscillate between negative and positive values, as can be seen from Fig. 9(c). However, the amplifier has smaller ringing and the ringing oscillation frequency is not constant and is not related to the measurement bit rate.

In summary, we must emphasize that bandwidth alone cannot be a complete measure to characterize the DDJ contributions of an LTI system. Although systems with small bandwidth tend to increase DDJ, step response or pulse response of the system is required to analyze the exact characteristics of output DDJ. The system can particularly be designed such that the samples of its pulse response are negligible at integer multiples of bit period to minimize DDJ. Along the same line of arguments and similar to Nyquist's zero-ISI pulse shaping [18], Huang et al. [8] and Gibby and Smith [25] have proposed channel pulse shapes that result in minimum jitter contributions from prior bits and, hence, optimize DDJ performance of the link.

In a communication link, if the channel response is not known or is time varying, zero-ISI pulse shaping is not possible. In such cases, an adaptive equalizer is utilized in the receiver to minimize ISI [19]. Similarly, if pulse shaping for the transmitted data sequence is not feasible due to channel unpredictability, a DDJ equalizer can be used in front of the clock recovery circuit.

\section{B. Integrated TIA}

To verify the validity of the DDJ prediction theory, we tested an integrated TIA. The TIA was implemented in a $0.18-\mathrm{mm}$ 
(a)

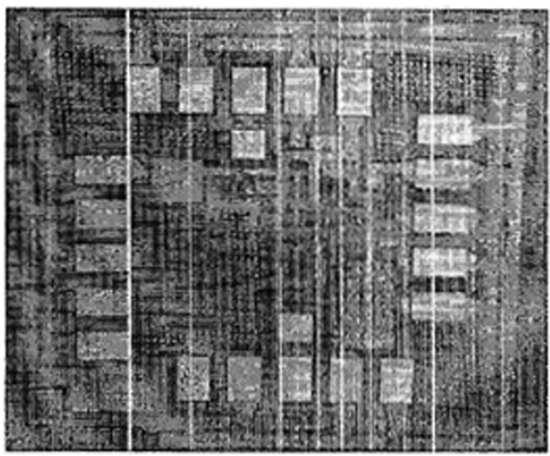

(b)

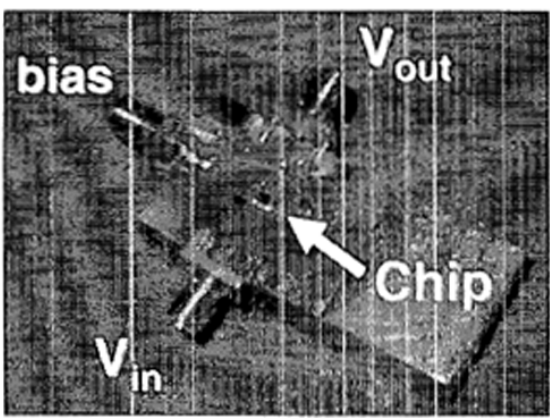

Fig. 10. (a) TIA die photograph. (b) Test board setup for the 10-Gb/s TIA

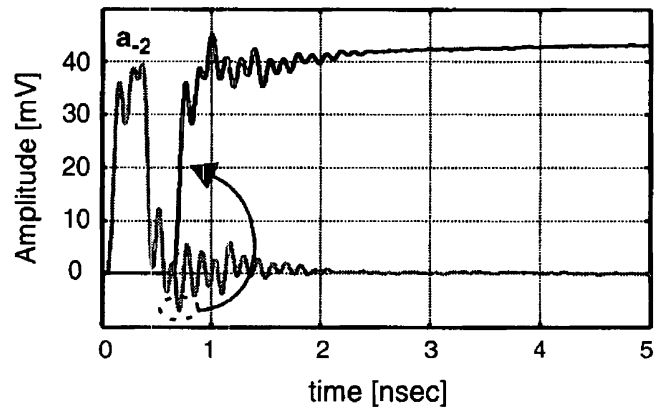

Fig. 11. TIA step response and impact of $a_{-2}$ pulse on $t_{0}$ in a " 101 " sequence at $3.3 \mathrm{~Gb} / \mathrm{s}$.

BiCMOS technology using only CMOS transistors and demonstrated a 9.2-GHz 3-dB bandwidth [26], [27]. We mount the amplifier on a brass substrate and build the additional circuitry around it on the same substrate using a low-loss Duroid PCB. The chip is wire bonded to microstrip transmission lines that then transfer the signal to subminiature A (SMA) connectors on the brass substrate. The die photograph and the test board setup are shown in Fig. 10. Although this TIA has enough bandwidth to operate at $10 \mathrm{~Gb} / \mathrm{s}$, the reflections from connectors and wire-bond mismatches in addition to the amplifier response cause the whole system to have a ringing step response as the measurement shows in Fig. 11. In spite of having enough bandwidth, the TIA along with the measurement setup exhibit a large amount of DDJ.

We measured DDJ of the TIA at two bit rates, i.e., 1.65 and $3.3 \mathrm{~Gb} / \mathrm{s}$, using the same procedure previously discussed. While the bit rates are within the bandwidth range of the TIA, we observed a significant amount of DDJ. The eye diagram at $1.65 \mathrm{~Gb} / \mathrm{s}$ is shown in Fig. 12(a). The measurement results are summarized in Table II. We should stress that the prediction of

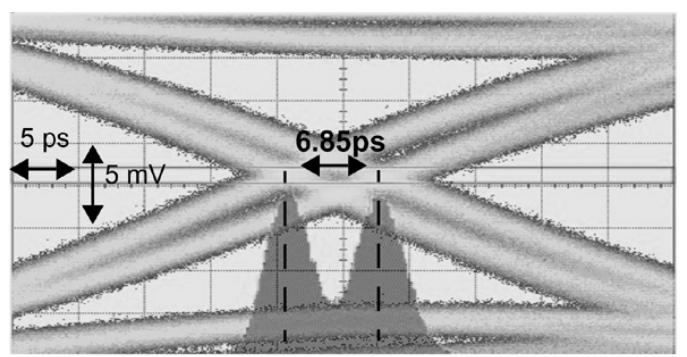

(a)

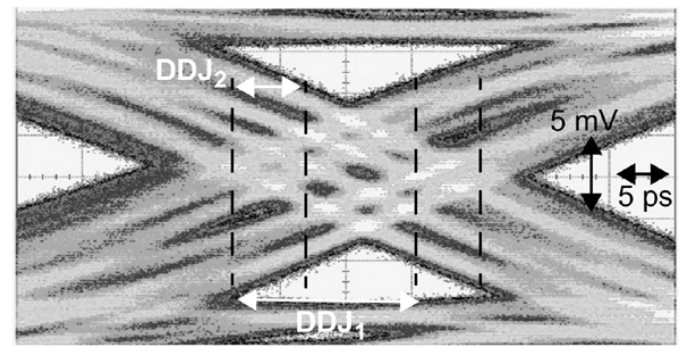

(b)

Fig. 12. TIA eye diagram when $\mathrm{DDJ}_{1}$ and $\mathrm{DDJ}_{2}$ are observable. (a) $1.65 \mathrm{~Gb} / \mathrm{s}$. (b) $3.37 \mathrm{~Gb} / \mathrm{s}$.

TABLE II

COMPARING MEASURED DD J ${ }_{1}$ AND PREDICTIONS OF ANALYTICAL EXPRESSION FOR THE 10-GB/s CMOS TIA

\begin{tabular}{c|c|c|c|c}
\hline Bit Rate & $\begin{array}{c}\text { Measured } \\
\text { DDJ }_{1}[\mathrm{psec}]\end{array}$ & Bit & $\begin{array}{c}\text { Predicted } \\
\Delta \boldsymbol{t}_{\boldsymbol{k}}[\mathrm{psec}]\end{array}$ & Error \\
\hline \hline $1.65 \mathrm{~Gb} / \mathrm{s}$ & 6.85 & $\mathrm{a}_{-2}$ & 6.8 & $0.85 \%$ \\
\hline $3.3 \mathrm{~Gb} / \mathrm{s}$ & 13.6 & $a_{-2}$ & 12.7 & $6.6 \%$ \\
\hline $3.37 \mathrm{~Gb} / \mathrm{s}$ & 14.1 & $a_{-2}$ & -12.4 & $12 \%$ \\
\hline $3.37 \mathrm{~Gb} / \mathrm{s}$ & $\mathrm{DDJ}_{2}=5.85$ & $a_{-3}$ & 5.7 & $2.5 \%$ \\
\hline
\end{tabular}

DDJ at several bit rates can be done with measuring the step response only once.

In the case of $1.65 \mathrm{~Gb} / \mathrm{s}$, DDJ prediction using the perturbation method has only $0.85 \%$ error. Larger scales of DDJ that are associated with prior bits with less dominant jitter contributions are often smaller than rms of RJ. Therefore, they are hard to measure or observe and are, thus, neglected. However, the perturbation method can still predict DDJ of larger scales. We measured the DDJ scale-one (DDJ ${ }_{1}$ ) and scale-two $\left(\mathrm{DDJ}_{2}\right)$ of the TIA at $3.37 \mathrm{~Gb} / \mathrm{s}$, where both were observable, as illustrated in Fig. 12. The measurement results are compared with the calculations in Table II. The perturbation method predicts scale-two DDJ with an accuracy of $2.5 \%$. The measured values of $\mathrm{DDJ}_{1}$ and $\mathrm{DDJ}_{2}$ are related to $\Delta t_{-2}$ and $\Delta t_{-3}$, as calculated from (13), respectively. The negative value of $\Delta t_{-2}$ corresponds to a negative shift in the zero crossing. In other words, all the sequences in which $\Delta t_{-2}$ is " 1 " will split from the zero crossings that occur at $t_{0}$ and will move to $t_{0}-\left|\Delta t_{-2}\right|$. On the other hand, positive $\Delta t_{-3}$ will split each crossing group to two groups, one remaining in the same position and one moving $\Delta t_{-3}$ to the right. Therefore, overall, four crossing groups can be observed, as in Fig. 12(b). 


\section{CONCLUSION}

DDJ is one type of DJ that results from residual effects of prior bits on a data threshold crossing time. It degrades BER and link performance as the data rates increase, while the system bandwidth budget is restricted. We have proposed a methodology to estimate a general LTI system's DDJ based on its step response. The method reduces the complexity remarkably because computation time grows linearly with the number of prior bits, whereas in conventional methods, complexity grows exponentially with the number of bits. We verified the validity of the analytical results with simulations and demonstrated experimentally that this approximation is reasonably accurate for several systems. In addition, we showed that certain pulse response shapes can result in a minimum peak-to-peak DDJ and can be used in designing the system. Finally, we highlighted that 3-dB bandwidth does not characterize DDJ of the system completely and the shape of the system step response is the important and essential element that determines DDJ characteristics.

\section{ACKNOWLEDGMENT}

The authors acknowledge Prof. H. Hashemi, University of Southern California, Los Angeles, A. Farajidana, California Institute of Technology, Pasadena, and M. Sharif, California Institute of Technology, for valuable discussions. The authors also thank A. Komijani and A. Natarajan, both of the California Institute of Technology, for providing feedback on this paper's manuscript.

\section{REFERENCES}

[1] "Fiber channel—Methodologies for jitter and signal quality specification-MJSQ," INCITS, Tech. Rep. REV 10.0, Mar. 10, 2003.

[2] A. Hajimiri, S. Limotyrakis, and T. H. Lee, "Jitter and phase noise in ring oscillators," IEEE J. Solid-State Circuits, vol. 34, no. 6, pp. 790-804, Jun. 1999.

[3] E. D. Sunde, "Self-timing regenerative repeaters," Bell Syst. Tech. J., vol. 36, no. 7, pp. 891-937, Jul. 1957.

[4] C. J. Byrne, B. J. Karafin, and D. B. Robinson, Jr., "Systematic jitter in a chain of digital regenerators," Bell Syst. Tech. J., vol. 42, no. 11, pp. 2679-2714, Nov. 1963.

[5] B. R. Saltzberg, "Timing recovery for synchronous binary data transmission,” Bell Syst. Tech. J., vol. 46, no. 3, pp. 593-622, Mar. 1967.

[6] Y. Takasaki, Digital Transmission Design and Jitter Analysis. Boston, MA: Artech House, 1991.

[7] F. M. Gardner, "Self-noise in synchronizers," IEEE Trans. Commun., vol. COM-28, no. 8, pp. 1159-1163, Aug. 1980.

[8] J. C. Y. Huang, K. Feher, and M. Gendron, "Techniques to generate ISI and jitter-free bandlimited Nyquist signals and a method to analyze jitter effects," IEEE Trans. Commun., vol. COM-27, no. 11, pp. 1700-1711, Nov. 1979.

[9] J. W. M. Bergmans, "Adaptive characterization of write-precompensation circuits," IEEE Trans. Magn., vol. 39, no. 4, pp. 2109-2114, Jul. 2003.

[10] M. Shimanouchi, "New paradigm for signal paths in ATE pin electronics are needed for serialcom device testing," in Proc. IEEE Int. Test Conf., Oct. 2002, pp. 903-912.

[11] M. Shimanouchi, "An approach to consistent jitter modeling for various aspects and measurement methods," in Proc. IEEE Int. Test Conf., Oct.-Nov. 2001, pp. 848-857.
[12] M. P. Li, J. Wilstrup, R. Jessen, and D. Petrich, "A new method for jitter decomposition through its distribution tail fitting," in Proc. IEEE Int. Test Conf., Sep. 1999, pp. 788-794.

[13] B. Analui, J. Buckwalter, and A. Hajimiri, "Estimating data-dependent jitter of a general LTI system from step response," presented at the IEEE MTT-S Int. Microwave Symp., Jun. 2005.

[14] A. Leon-Garcia, Probability and Random Processes for Electrical Engineering, 2nd ed. Reading, MA: Addison-Wesley, 1994.

[15] Y. Cai, S. A. Werner, G. J. Zhang, M. J. Olsen, and R. D. Brink, "Jitter testing for multi-gigabit backplane Serdes-techniques to decompose and combine various types of jitter," in Proc. IEEE Int. Test Conf., Oct. 2002, pp. 700-709.

[16] J. Wilstrup, "A method of serial data jitter analysis using one-shot time interval measurements," in Proc. IEEE Int. Test Conf., Oct. 1998, pp. 819-823.

[17] J. Buckwalter, B. Analui, and A. Hajimiri, "Data-dependent jitter and crosstalk-induced bounded uncorrelated jitter in copper interconnects," in IEEE MTT-S Int. Microwave Symp. Dig., vol. 3, Jun. 2004, pp. $1627-1630$.

[18] H. Nyquist, "Certain topics in telegraph transmission theory," AIEE Trans., vol. 47, pp. 617-644, Apr. 1928.

[19] J. G. Proakis, Digital Communications, 4th ed. Boston, MA: McGrawHill, 2001.

[20] J. Buckwalter, B. Analui, and A. Hajimiri, "Predicting data-dependent jitter," IEEE Trans. Circuits Syst. II, Exp. Briefs, vol. 51, no. 9, pp. 453-457, Sep. 2004.

[21] R. Bellman, Perturbation Techniques in Mathematics, Physics, and Engineering. New York: Holt, Rinehart and Winston, 1964.

[22] A. B. Carlson, Communication Systems, 3rd ed. Boston, MA: McGraw-Hill, 1986.

[23] W. R. Bennett, "Statistics of regenerative digital transmission," Bell Syst. Tech. J., vol. 37, pp. 1501-1542, Nov. 1958.

[24] G. L. Cariolaro and F. Todero, "A general spectral analysis of time jitter produced in a regenerative repeater," IEEE Trans. Commun., vol. COM-25, no. 4, pp. 417-426, Apr. 1977.

[25] R. A. Gibby and J. W. Smith, "Some extensions of Nyquist's telegraph transmission theory," Bell Syst. Tech. J., vol. 44, pp. 1487-1510, Sep. 1965.

[26] B. Analui and A. Hajimiri, "Multi-pole bandwidth enhancement technique for trans-impedance amplifiers," in Proc. Eur. Solid-State Circuits Conf., Sep. 2002, pp. 303-306.

[27] B. Analui and A. Hajimiri, "Bandwidth enhancement for transimpedance amplifiers," IEEE J. Solid-State Circuits, vol. 39, no. 8, pp. 1263-1270, Aug. 2004.

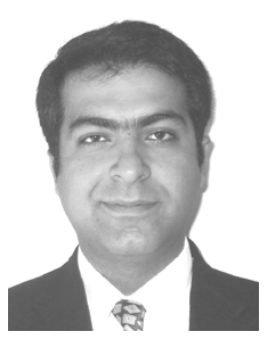

Behnam Analui (S'97) received the B.S. and M.S. degrees in electronics engineering from the Sharif University of Technology (SUT), Tehran, Iran, in 1998 and 2000, respectively, and is currently working toward the Ph.D. degree at the California Institute of Technology (Caltech), Pasadena.

His research interest is high-speed integrated-circuit design for wireline communications. During Summer 2003, he was with the Mixed-Signal Communications Integrated Circuit (IC) Design Group, IBM T. J. Watson Research Center, Yorktown Heights, NY, where he designed a data quality monitoring circuit for multimode fiber adaptive equalization. He is currently with Luxtera Inc., Carlsbad, CA.

Mr. Analui was the recipient of the 1994 Silver Medal of the National Mathematics Olympiad, the 2000 SUT Presidential Honorary Award as the chair of Technical Program Committee in International Millennium Seminar on Electrical Engineering, the 2000 California Institute of Technology Atwood Fellowship, and the 2002 Analog Devices Outstanding Student Designer Award. 


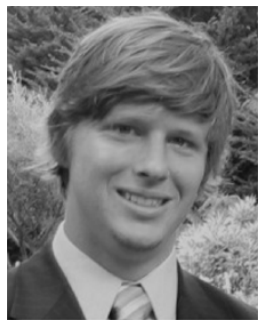

James F. Buckwalter (S'01) received the B.S. degree in electrical engineering from the California Institute of Technology, Pasadena, in 1999, the M.S. degree in electrical engineering from the University of Santa Barbara, in 2001, and Ph.D. degree in electrical engineering from the California Institute of Technology, in 2005. His doctoral dissertation concerned problems relating to timing jitter in high-speed communications and testing.

From 1999 to 2000, he was a Research Scientist with Telcordia Technologies, where he was involved with rate-agile burst-mode electronics under a Next-Generation Internet Defense Advanced Research Projects Agency (DARPA) project. While with the University of California at Santa Barbara, he developed coupled phase-locked loops for microwave beam steering. In Summer 2004, he was with the IBM T. J. Watson Research Center, Yorktown Heights, NY.

Dr. Buckwalter was the recipient of a 2004 IBM Ph.D. Fellowship.

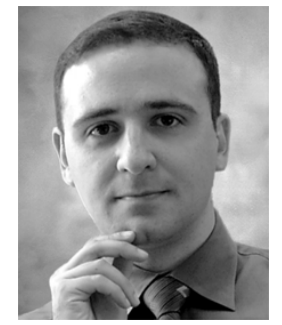

Ali Hajimiri (S'95-M'99) received the B.S. degree in electronics engineering from the Sharif University of Technology, Tehran, Iran, in 1994, and the M.S and $\mathrm{Ph} . \mathrm{D}$. degrees in electrical engineering from Stanford University, Stanford, CA, in 1996 and 1998, respectively.

From 1993 to 1994, he was a Design Engineer with Philips Semiconductors, where he was involved with a BiCMOS chipset for global system for mobile communications (GSM) and cellular units. In 1995, he was with Sun Microsystems, where he was involved with the UltraSPARC microprocessor's cache RAM design methodology. During Summer 1997, he was with Lucent Technologies (Bell Laboratories), Murray Hill, NJ, where he investigated low phase-noise integrated oscillators. In 1998, he joined the Faculty of the California Institute of Technology, Pasadena, where he is currently an Associate Professor of electrical engineering and the Director of Microelectronics and Noise Laboratories. $\mathrm{He}$ is a cofounder of Axiom Microdevices Inc. He coauthored The Design of Low Noise Oscillators (Boston, MA: Kluwer, 1999). He holds several U.S. and European patents. His research interests are high-speed and RF integrated circuits. He was a Guest Editorial Board member of the Transactions of the Institute of Electronics, Information and Communication Engineers of Japan (IEICE).

Dr. Hajimiri is an associate editor for the IEEE JOURNAL OF SOLID-STATE CIRCUITS. He is a member of the Technical Program Committee of the International Solid-State Circuits Conference (ISSCC). He has also served as an associate editor for the IEEE TRANSACTIONS ON CIRCUITS AND SYSTEMS-Part II: ANALOG AND DigitAl SignAL PROCESSING. He is a member of the Technical Program Committees of the International Conference on Computer-Aided Design (ICCAD). He was a guest editor for the IEEE TRANSACTIONS ON MicROWAVE THEORY AND TECHNIQUES. He is listed on the Top 100 Innovators (TR100) List. He was the recipient of the Gold Medal of the National Physics Competition and of the Bronze Medal of the 21st International Physics Olympiad, Groningen, The Netherlands. He was a corecipient of the ISSCC 1998 Jack Kilby Outstanding Paper Award and a three-time recipient of the IBM Faculty Partnership Award, as well as the National Science Foundation (NSF) CAREER Award. 